\title{
Reconsidering Scale and Scaling in CSCW Research
}

\author{
Chiara Rossitto \\ Stockholm University \\ Kista, Sweden \\ chiara@dsv.su.se
}

\section{Susanne Bødker}

Aarhus University

Aarhus, Denmark

bodker@cs.au.dk

\section{Katie Berns}

Stockholm University

Kista, Sweden

katie@dsv.su.se

\author{
Airi Lampinen \\ Stockholm University \\ Kista, Sweden \\ airi@dsv.su.se
}

\author{
Ann Light \\ ann.light@gmail.com

\section{Julie Hui} \\ University of Michigan \\ Michigan, USA \\ juliehui@umich.edu
}

University of Sussex, Malmö University

Brighton, UK, Malmö, Sweden

\section{ABSTRACT}

This one-day workshop invites discussion on the various socio-technical processes and dynamics that characterize scale and scaling in local, community-sited initiatives. Seeking to move beyond a view of scale as mere growth in numbers and a matter of technology-mediated replication, the workshop aims at developing a nuanced vocabulary to talk about various forms of scale and practices of scaling in CSCW research. It will bring together interdisciplinary scholars, activists, practitioners and representatives of the public sector who wish to question and further develop the notion of

Permission to make digital or hard copies of all or part of this work for personal or classroom use is granted without fee provided that copies are not made or distributed for profit or commercial advantage and that copies bear this notice and the full citation on the first page. Copyrights for components of this work owned by others than ACM must be honored. Abstracting with credit is permitted. To copy otherwise, or republish, to post on servers or to redistribute to lists, requires prior specific permission and/or a fee. Request permissions from permissions@acm.org.

CSCW'20 Companion, October 17-21, 2020, Virtual Event, USA

(C) 2020 Association for Computing Machinery.

ACM ISBN 978-1-4503-8059-1/20/10...\$15.00

https://doi.org/10.1145/3406865.3419409 
scale generally associated with processes of upscaling. The workshop provides a forum to discuss: $i$ ) concepts, theories and empirical cases that broaden our view of what constitutes scale; and ii) the implications for CSCW research in assessing the long-term impact and sustenance of socio-technical innovations. The workshop will accommodate up to twenty participants and will be run virtually.

\section{KEYWORDS}

scale; scaling; local initiatives; theories, case studies; sociotechnical systems.

\section{ACM Reference Format:}

Chiara Rossitto, Airi Lampinen, Susanne Bødker, Ann Light, Katie Berns, and Julie Hui. 2020. Reconsidering Scale and Scaling in CSCW Research. In Companion Publication of the 2020 Conference on Computer Supported Cooperative Work and Social Computing (CSCW'20 Companion), October 17-21, 2020, Virtual Event, USA. ACM, New York, NY, USA, 9 pages. https://doi.org/10.1145/3406865.3419409

\section{INTRODUCTION}

The problem of scale is not new to $\mathrm{CSCW}$ and $\mathrm{HCl}$ research. Scholars have, for instance, addressed the challenges, associated with social networking sites and big data, to manage millions of users across devices and contexts of use [2]. Nevertheless, community-led and grassroots projects, civic and collaborative economy initiatives, as well as research on sustainability and sustainable futures problematize the very notion of growth as a simple matter of attracting ever larger numbers of users, or replicating (i.e cloning) initiatives through the use of digital platforms. While mainstream, global platforms (e.g. Uber, Airbnb) strive for expansion as a part of their business model, grassroots initiatives might have different aspirations, concerns and economic constraints that reflect on the ways they "move forward", find their ways across contexts and establish collaborations with third actors - from people to formal organizations. This poses conceptual, methodological, analytical and design challenges which we set out to address through this workshop.

We will unpack the different dimensions along which scaling can occur and the various forms it can take. Through focused and in-depth discussions, we aim at shifting from a focus on scale (intended as a mere quantitative growth) to one on scaling - that is, the variety of practices, along with the role of human and non-human agents, that contribute to the ways local initiatives proliferate across contexts and over time. This, we argue, is paramount to: i) question the implicit assumptions that socio-technical projects can smoothly be expanded without changing their organization and framings (see [11]); ii) recuperate non-scalable initiatives, that is, projects that are not necessarily equipped (or aspiring) to attract large numbers of participants, but are, nevertheless, locally relevant and impactful.

Our workshop provides opportunities for community building and the development of future research and design directions. It is structured around creating a shared agenda for CSCW research and further addressing the diverse socio-technical dynamics underlying scaling. 


\section{Conceptualizing scale and scaling}

Research from a range of academic disciplines has begun to look into different upscaling processes. Work within transition studies [9] has distinguished, for instance, between growing, replication, accumulation and transformation. Growing and replication, respectively, relate to an increased number of actors participating in a given initiative and reusing the same concept in different locations. Accumulation and transformation are, instead, indicative of more qualitative changes: in the first, different initiatives are connected to each other, in the latter a given initiative shapes change at an institutional level. Intersecting research on design and social innovation, Manzini [8] has suggested two scaling strategies. Scaling out, consists of recreating and reconfiguring horizontally the most promising practices across contexts. This strategy entails the replication of a project, while attuning it to a new context. The second strategy, scaling up, deals with connecting and integrating several small collaborative projects and grassroots initiatives into larger framework programs. Relatedly, $\mathrm{HCl}$ research [1] has drawn attention to different modes of scaling. Sustaining relates to the work of organizing initiatives (e.g. defining practices, attracting members and resources). Growing includes processes to build up and consolidate the socio-technical infrastructure to enable more people to take part. Spreading deals with the creation and dissemination of new skills, ideas and knowledge.

Previous work has also investigated the relationships grassroots initiatives establish with local actors, including formal organizations, to consolidate their vision and achieve impact. This research [3] has characterized building anew as the strategy whereby communities can grow by collaborating with local actors or tapping in existing infrastructure. Their work has shown, however, that both members' sense of ownership and their experience of the role of the organization might change throughout such processes. Gounding their discussion in the case of Hoffice - a self-organizing network experimenting with an alternative social model for collectively organizing flexible forms of work in private homes ( see [10] for details) - Lampinen and Rossitto [5] have discussed the challenges of scaling out and replicating Hoffice in other contexts such as public libraries. This work shows that core visions and ideas might be entirely transformed, to the extent that they become something else, when initiatives are replicated by - and for - third-party actors that fail (or purposefully refuse) to appropriate initiatives' original values and motivations to organize. Overall, this research shows that scaling out encompasses not only adapting technologies and practices to new settings, but also the (re)negotiation and transformation of core values.

\section{WORKSHOP THEMES: SCALE AND SCALING IN CSCW RESEARCH}

This workshop intends to develop accounts of scaling for CSCW research that encompass different backgrounds and perspectives. Contributors may wish to address a range of themes including, but not restricted to: 
Conceptual aspects. What alternative concepts and terminology can be adopted to indicate that scaling community-centered initiatives is not necessarily a form of technology-mediated replication? How can we trouble the assumption that growing and growth are always desirable strategies and goals, when this seems not to be the case for local initiatives? Our previous work [5], for instance, has pointed to scaling down as the explicit design strategy to organize at a smaller - rather than bigger - scale and to use digital platforms to connect small subgroups to the broader local initiative as a way to manage growth. Relatedly, Light and Clodagh [7] have suggested meshing as a concept to describe how physical places and people can be connected by creating socio-technical infrastructures for sharing locally - as opposed to global platforms that homogenize interactions and promote crisp transactions. Under this theme, we invite participants to share i) experiences of adopting alternative conceptualizations in their research and ii) propose potential vocabularies to diversify how we conceive of scaling in CSCW research.

Analytical aspects. Moving beyond a view of scale as the reproduction of technology-mediated practices opens to empirical and theoretical understandings of the ways local initiatives broaden their activities and put key ideas into circulation. We see potential here to investigate the role of human mediation in putting personal experiences and previous projects' outcomes into circulation, thus shaping the proliferation of related initiatives. Moreover, we invite contributions exploring the ways local projects become interconnected over time, and events add up to more than just a series of isolated happenings unfolding over time [6]. Addressing these issues encompasses an understanding of: $i$ ) the ways different elements of socio-technical interventions - e.g. roles, know-how and professional skills, practices, relationships and attachments, digital and physical infrastructure, values and ideas - are moved on to other projects and socio-cultural settings; ii) when, and whether, the transformation of one of them may result in the creation of another initiative altogether.

(Inter)organizational aspects. When considering how grassroots initiatives may proliferate and morph over time, issues of scale and long-term sustenance become entangled with questions of institutionalization, be it in the form of partnerships or formalizing the initiative itself into a different type of an organization. Working from the understanding that not all initiatives aspire to become scalable profit-seeking ventures, we see a number of open questions regarding engagement with and/or transformation into alternative organizational forms (public organizations, NGOs, cooperatives, etc.) so as to support the longer-term sustenance of (the aims of) grassroots initiatives. In particular, we call for careful attention to how grassroots initiatives (may) morph as they collaborate, or even merge, with formal organizations - a process that requires navigating tensions in values, work practices, ways of using technology, and visions for what constitutes desirable impact. We invite participants to explore the processes and dynamics underlying such (inter)organizational transformation, and the challenges that arise when the grassroots meets the structure [4]. Under this theme, participants may address 
issues such as: $i$ ) the role of existing infrastructures in enabling and mediating such transformation processes; ii) the situated factors and circumstances crucial for specific collaborations and transformations to occur - or not; iii) what the various actors involved are positioned to enable as well as how their aspirations and concerns are reconfigured through (inter)organizational transformation.

\section{WORKSHOP GOALS}

The goals for the workshop include:

- Identifying research areas, theories and empirical cases that help expand accounts of what constitutes scale and scaling;

- Developing a vocabulary to talk about different forms of scale;

- Highlighting the various dimensions along which scaling processes and dynamics can be articulated and understood;

- Discussing the role of digital technologies and existing infrastructures in enabling, or hindering, scaling processes;

- Discussing the relationships between a multifaceted notion of scale and impact. Can the circulation of an idea be regarded as an indication of impact despite the different ways of organizing and mobilizing work between local initiatives?

- Outlining elements of socio-technical interventions that are challenging to replicate;

- Unpacking the connections between bottom-up initiatives and public organizations and their relevance in providing CSCW research with more nuanced accounts of scaling;

- Encouraging interactions and collaborations between researchers, practitioners and activists for whom aspects of scale are central to mobilize and sustain action over time;

- Supporting and scaffolding collaborations beyond the time frame of the workshop.

\section{TARGET AUDIENCE}

This one-day workshop is targeted at researchers, practitioners, and activists interested in issues of scale and scaling, with a particular focus on grassroots initiatives and their connections to civic and public organizations. We plan to accept 20 participants at maximum.

\section{WORKSHOP ACTIVITIES}

The workshop will entail a variety of activities enabling in-depth discussion and community building. Considering the constraints of running a workshop virtually, and across time zones, we plan to organize this one-day workshop in the form of two half-day sessions of about 3 hours each. This is 
intended to promote broader and more diverse participation as well as to mitigate the exhaustion related to video-mediated collaborative activity.

\section{Day 1 - Session 1}

Introduction - 20 minutes

The organizers will open the workshop by introducing the agenda and goals for the two days. They will facilitate a lightning round of introductions, giving each participant a moment to introduce themselves and their interest in the topic.

Presentation - 80 minutes

Participants will introduce their work with compact, 4-minute talks. They will be asked to clarify what influences their particular perspective(s) on scaling. This will be instrumental to foster and broaden shared understandings of how we can leverage different views on scale, and to start identifying main similarities and differences in the use of the concept across disciplines and empirical settings. A shared GoogleDoc will be set up to support discussion and collaborative note-taking.

Break - 20 minutes

Mapping scale - 60 minutes

Based on emerging themes and shared interests, participants will work in breakout groups to develop mind maps that visualize the variety of concepts and theories, analytical foci, and empirical examples of technology-mediated practices that can broaden our understandings of scale. Miro whiteboards will be used for these activities. The outcomes of each group discussion will be shared with the rest of the participants. Wrapping up the day and final remarks - 15 minutes

Day 2 - Session 2

Introduction - 15 minutes

The organizers will open this second session by summarizing the previous day's activities and outcomes. We plan for a quick round of introductions, should additional people join this session.

Collaborative writing - 45 minutes

Building on the mind maps, workshop attendees will work in breakout groups to draft future research questions, empirical studies and technological designs reflecting the various scaling mechanisms discussed. Based on the participants' interests, this activity might also serve as a starting point for a blogpost or an Interactions piece.

Break - 20 minutes

Wrapping up and plans for the future - 40 minutes

After summarizing the workshop's outcomes, the organizers will facilitate a discussion to brainstorm possible collaborations and future plans.

Informal discussion and socializing - 60 minutes

This last hour is intended to allow participants to sit back and relax, while still engaging with the other colleagues. This last phase will be an opportunity to network and socialize. 


\section{EQUIPMENT NEEDS}

The workshop has no equipment needs beyond the usual. We plan to host the workshop over Zoom, or a similar platform in line with workshop chairs' recommendations. We will also use further tools, such as Padlet, GoogleDocs, and Miro to encourage collective note-taking and the creation of other working contents.

\section{PRE-WORKSHOP PLANS}

Participant Recruitment. Participants will be recruited from the $\mathrm{CSCW}$ and $\mathrm{CHI}$ communities, and from the extended research networks of the organizers. Detailed information about the workshop will be made available at our workshop website. We will reach out to international, interdisciplinary networks, such as the SharingAndCaring COST Action (http://sharingandcaring.eu/), EUSSET, and The Community of Sociotechnical Systems Researchers (https://sociotech.net/). We will circulate the call through relevant mailing lists and social media. Given that the workshop will be virtual, we will look at this as an opportunity to broaden participation, extending the invitation to researchers, practitioners, activists, representatives of the public sectors, based across countries and continents. This will be essential to leverage a diverse, multi-party perspective on scale and scaling.

Submissions and participant selection. Authors are invited to submit a 2-4 page position paper in the Legacy SIGCHI Extended Abstracts Format (Word / LaTeX). However, to promote broader participation (including but not limited to activists, representatives from the public sector, and practitioners in the industry), we offer the option of submitting alternative material in the form of design portfolios, short manifestos, or the like. We encourage potential participants to discuss their interest in the workshop themes, welcoming reports of (preliminary) empirical results, theoretically oriented pieces, or short statements regarding next steps of working on a topic. Submissions will be reviewed by the workshop organizers. Participants will be selected based on the relevance of their submissions and their potential to spark discussion. Accepted submissions will be made available to participants before the event as well as posted on the workshop website with participants' consent.

We will do our best to accommodate specific, accessibility needs, and while we cannot promise to solve every issue, we will try our best. Authors will be invited to specify particular requirements in their position papers or by directly contacting the organizers.

Workshop website. Should the proposal be accepted, a website will be created to advertise the event, share material among participants, as well as to document and disseminate workshop outcomes. 


\section{POST-WORKSHOP PLANS}

The key objectives of the workshop are to build community and foster collaborations among participants, and we will discuss possible next steps to further the work together. These could include the organization of future events, writing a piece for Interactions, or maybe collaborating on a special issue or an edited book. Our aim here is to facilitate initiative from participants and, as such, we remain open to different outputs that align with participants' needs and interests.

\section{ORGANIZERS}

Chiara Rossitto is Assistant Professor of $\mathrm{HCl}$ at Stockholm University. Her research has problematized the role of technology in enabling people's involvement in socio-political debates, scaling and sustaining community-led initiatives over time. Her ongoing work investigates collective, technologymediated initiatives of care for the environment.

Airi Lampinen is an Associate Professor in $\mathrm{HCl}$ at Stockholm University and a Docent in Social Psychology at the University of Helsinki. She has studied interpersonal dynamics in peer-to-peer exchange extensively. Her ongoing research focuses on interpersonal challenges in sharing economies and alternative, member-driven peer-to-peer initiatives.

Susanne Bødker is Professor of $\mathrm{HCl}$ at the Department of Computer Science, Aarhus University. She works with activity theoretical HCl, Participatory Design and Computer Supported Cooperative Work. She is currently working on her ERC Advanced research project Common Interactive Objects, that takes a new theoretical focus on how we collaborate and make sense of the interactive objects in our everyday lives.

Ann Light is Professor of Design and Creative Technology at the University of Sussex in the UK and Professor at Malmo University in Sweden. She specializes in the social impact of technology, and particularly the deployment of platforms. Her design work concerns innovation in social process, social justice and sustainability, researched using participatory methods.

Katie Berns is a PhD student in $\mathrm{HCl}$ at Stockholm University. Her research is in Digital Civics and Sustainable Interaction Design with a focus on the design of ICT for civic participation and community-led initiatives within the domain of food system sustainability.

Julie Hui is an incoming Assistant Professor at the University of Michigan School of Information. She researches how technology influences workforce development, and visa versa. She has worked with various online and offline communities, including gig workers, under-resourced entrepreneurs, makerspaces, and crowdfunders. 


\section{ACKNOWLEDGMENTS}

This work has been supported by Swedish Research Council grant number 2017-05382-3, the Kamprad Family Foundation grant number 20200087, and the COST Action CA16121 From Sharing to Caring.

\section{REFERENCES}

[1] Aksel Biørn-Hansen and Maria Håkansson. 2018. Building Momentum: Scaling up Change in Community Organizations. In Proceedings of the 2018 CHI Conference on Human Factors in Computing Systems (CHI '18). Association for Computing Machinery, Montreal QC, Canada, 1-13. https://doi.org/10.1145/3173574.3173984

[2] Barry Brown, Susanne Bødker, and Kristina Höök. 2017. Does $\mathrm{HCl}$ scale? scale hacking and the relevance of $\mathrm{HCl}$. Interactions 24, 5 (Aug. 2017), 28-33. https://doi.org/10.1145/3125387

[3] Susanne Bødker, Henrik Korsgaard, and Joanna Saad-Sulonen. 2016. 'A Farmer, a Place and at least 20 Members' The Development of Artifact Ecologies in Volunteer-based Communities. In Proceedings of the 19th ACM Conference on Computer-Supported Cooperative Work \& Social Computing (CSCW '16). Association for Computing Machinery, San Francisco, California, USA, 1142-1156. https://doi.org/10.1145/2818048.2820029

[4] Andy Dow, Rob Comber, and John Vines. 2019. Communities to the left of me, bureaucrats to the right... here I am, stuck in the middle. Interactions 26, 5 (Aug. 2019), 26-33. https://doi.org/10.1145/3351735

[5] Airi Lampinen, Chiara Rossitto, and Christofer Gradin Franzén. 2019. Scaling Out, Scaling Down: Reconsidering growth in grassroots initiatives. In ETHNOCOL 2019. Edinburgh.

[6] Jay L. Lemke. 2000. Across the Scales of Time: Artifacts, Activities, and Meanings in Ecosocial Systems. Mind, Culture, and Activity 7, 4 (Nov. 2000), 273-290. https://doi.org/10.1207/S15327884MCA0704_03 Publisher: Routledge _eprint https://doi.org/10.1207/S15327884MCA0704_03.

[7] Ann Light and Clodagh Miskelly. 2019. Platforms, Scales and Networks: Meshing a Local Sustainable Sharing Economy. Computer Supported Cooperative Work (CSCW) 28, 3 (June 2019), 591-626. https://doi.org/10.1007/s10606-019-09352-1

[8] Ezio Manzini. 2015. Design, When Everybody Designs. The MIT Press.

[9] Rolf Naber, Rob Raven, Matthijs Kouw, and Ton Dassen. [n.d.]. Scaling up sustainable energy innovations | Elsevier Enhanced Reader. https://doi.org/10.1016/j.enpol.2017.07.056

[10] Chiara Rossitto and Airi Lampinen. 2018. Co-Creating the Workplace: Participatory Efforts to Enable Individual Work at the Hoffice. Computer Supported Cooperative Work (CSCW) 27, 3 (Dec. 2018), 947-982. https://doi.org/10.1007/s 10606018-9319-z

[11] Anna Lowenhaupt Tsing. 2015. The Mushroom at the End of the World: On the Possibility of Life in Capitalist Ruins. Princeton University Press. 